\title{
Quelques facteurs explicatifs de l'augmentation de l'excrétion azotée fécale observée avec les fourrages pauvres traités à l'ammoniac
}

\author{
L Hassen, M Chenost, B Michalet-Doreau \\ INRA Theix, station de recherches sur la nutrition des herbivores, \\ 63122 Saint-Genès-Champanelle, France
}

\begin{abstract}
Summary - Some factors to explain the high non digestible fecal nitrogen with ammonia treated forages. The increase, after ammonia treatment, of the non digestible nitrogen content of a straw, a mature hay and a maize stover is essentially composed of soluble $N$, which suggests inefficient use of the added nitrogen by the rumen microorganisms.
\end{abstract}

Le traitement des pailles à l'ammoniac $\left(\mathrm{NH}_{3}\right)$ entraîne une augmentation anormale de leur teneur en matières azotées non digestibles (MAND) (Benahmed et Dulphy, 1985). Or, l'essentiel de l'azote fixé serait très rapidement dégradé dans le rumen (Michalet-Doreau et Guedes, 1989). L'objet de ce travail est de mesurer, sur les mêmes fourrages, traités ou non à l'ammoniac, la teneur en MAND, la dégradabilité ruminale de l'azote et les parts respectives de I' $\mathrm{N}$ alimentaire et microbien dans les fèces.

Matériel et méthodes - Nous avons utilisé 3 fourrages : une paille de blé $(P)$, un foin de dactyle/ray-grass récolté tardivement (F) et des tiges de mais (TM), en l'état (NT) ou traités à l'ammoniac $(A)$ à raison de, respectivement, $5,0,1,6$ et $3,0 \mathrm{~kg}$ d' $\mathrm{NH}_{3}$ pour $100 \mathrm{~kg}$ brut de fourrage.

Essai 1 - Trois lots de 6 moutons mâles castrés $(52 \mathrm{~kg})$ en cage à digestibilité ont reçu ces 6 fourrages en quantité limitée (30 g. kg PV ${ }^{-0.75} \cdot \mathrm{j}^{-1}$ ). On a dosé l'N total, I'N soluble dans un tampon phosphate à $\mathrm{pH} 6,9$ et l' $N$ fixé au NDF (N-NDF) sur les fourrages et sur les fèces fraîches lyophilisées, ainsi que l'ARN des fèces (Ushida et al, 1985) pour estimer la part d' $\mathrm{N}$ microbien dans ces fèces.

Essai 2 - Par ailleurs, 3 vaches fistulées du rumen et recevant $7,0 \mathrm{~kg}$ d'un régime constitué de foin et de concentré (70/30) ont été utilisées pour mesurer la cinétique de dégradation in sacco et la dégradabilité (DT) de I'N des 6 fourrages dans le rumen. La colonisation bactérienne des résidus de sachets a été réduite en utilisant la technique de battage au stomacher, et la fraction d' $\mathrm{N}$ bactérien restant fixé sur les sachets après lavage a été prise en compte au niveau du calcul de I'N résiduel.

Essai 3 - On a utilisé 3 moutons $(60 \mathrm{~kg})$ fistulés du duodénum et de l'iléon (canule réentrante) et recevant $1,4 \mathrm{~kg}$ du régime distribué aux vaches, pour estimer la part d'N microbien dans les fèces provenant d'une éventuelle refermentation dans le gros intestin. Pour cela, après avoir séjourné $16 \mathrm{~h}$ dans le rumen, les résidus de paille traitée ou non ont été mis dans des microsachets mobiles $(0,5 \mathrm{~g}, 2 \times 2 \mathrm{~cm})$, introduits dans le duodénum et récupérés au niveau iléal ou fécal. On a dosé sur les résidus de sachets I'N total et I'ARN microbien. 
Réss Itats et discussion - Le traitement des fourrages à l' $\mathrm{NH}_{3}$ a augmenté l'excrétion fécale azotée d'environ $50 \%$ (tableau I). Bien que l'azote apporté par le traitement (tableau I) soit rapidement (plus de $70 \%$ en $2 \mathrm{~h}$ ) et presque entièrement (plus de $80 \%$ ) dégradé dans le rumen, l'augmentation des MAND est essentiellement constituée d'azote soluble, au moins pour F et TM, alors que la contribution de l'N-NDF à l'augmentation des MAND est faible, voire nulle (tableau I). La fixation d'une partie de l' $\mathrm{N}$ du traitement sur les parois indigestibles du fourrage reste donc faible, contrairement aux résultats rapportés par Mason et al (1989). Enfin, l'azote bactérien ne contribue que pour 7 à $23 \%$ à l'augmentation des MAND. La refermentation des fourrages dans le gros intestin reste donc peu importante, ce qui est confirmé par la faible aug- mentation du \% d'N bactérien entre I'IG et le GI (tableau I).

Ces résultats, complétant ceux de Ramihone (1987), montrent qu'une fraction importante de I'N apporté par le traitement est inutilisée par les microbes du rumen et est excrétée dans les fèces. Cet $N$ est pourtant en grande partie libéré dans le rumen et se retrouve sous forme d'N soluble dans les fèces.

Benahmed H, Dulphy JP (1985) Ann Zootech 34, 335-346

Mason VC, Cook JE, Cooper EM, Hoadley CJ, Cocksburn JE (1989) Feed Sci Technol 24, 313-326

Michalet-Doreau B, Guedes CVM (1989) Ann Zootech 38, 259-268

Ramihone $B$ (1987) Thèse de doctorat, université des sciences et techniques du Languedoc, Montpellier, $85 \mathrm{p}$

Ushida K, Lassalas B, Jouany JP (1985) Reprod Nutr Dév 25, 1037-1046

Tableau I. Dégradation de I'N alimentaire dans le rumen, contribution des formes soluble, alimentaire et microbienne à l'augmentation de l' $\mathrm{N}$ fécal, et estimation de la synthèse d' $\mathrm{N}$ microbien dans le $\mathrm{Gl}$, à la suite du traitement à l' $\mathrm{NH}_{3}$ d'une paille, d'un foin et de tiges de maïs.

\begin{tabular}{|c|c|c|c|c|c|c|c|c|c|}
\hline & \multicolumn{3}{|c|}{ Foin } & \multicolumn{3}{|c|}{ Tiges de maïs } & \multicolumn{3}{|c|}{ Paille } \\
\hline & $N T$ & $A$ & $A-N T$ & $N T$ & $A$ & $A-N T$ & $N T$ & $A$ & $A-N T$ \\
\hline \multicolumn{10}{|l|}{$\mathrm{N}$ alimentaire } \\
\hline Dég $2 \mathrm{~h}^{\mathrm{a}}$ & 27,5 & 52,2 & 79,0 & 47,3 & 72,2 & 88,0 & 46,0 & 55,3 & 71,0 \\
\hline $\mathrm{DT}^{\mathrm{a}}$ & 52,1 & 70,7 & 91,0 & 59,5 & 80,6 & 94,0 & 56,3 & 66,9 & 83,0 \\
\hline \multicolumn{10}{|l|}{$\mathrm{N} \times 6,25$ fécal } \\
\hline $\begin{array}{l}\text { Quantité } \\
\text { Composition }\end{array}$ & 43,0 & 65,0 & 22,0 & 43,0 & 66,0 & 23,0 & 39,0 & 55,0 & 16,0 \\
\hline $\begin{array}{l}N \text { sol } \\
N-N D F \\
N \text { micr }\end{array}$ & 38,8 & 54,5 & $\begin{array}{c}71,4 \\
\text { nul } \\
6,6\end{array}$ & 11,2 & 35,0 & $\begin{array}{c}79,6 \\
\text { nul } \\
23,4\end{array}$ & 27,2 & 26,9 & $\begin{array}{l}26,3 \\
15,7 \\
11,3\end{array}$ \\
\hline \multicolumn{10}{|c|}{$\mathrm{N}$ bactérien $\% \mathrm{~N}$ des résidus des sachets } \\
\hline Rumen $16 \mathrm{~h}$ & $(v$ & & & & & & 52,0 & 36,0 & \\
\hline Intestin grêle & $(\mathrm{mc}$ & & & & & & 3,2 & 2,0 & \\
\hline Gros intestin & (mc & & & & & & 5,2 & 3,6 & \\
\hline
\end{tabular}

${ }^{a} \mathrm{~N}$ dégradé au bout de $2 \mathrm{~h}$ (\% $\mathrm{N}$ initial) et $D T$ de $\mathrm{IN}$ dans le rumen; ${ }^{b}$ exprimée en $\mathrm{g} / \mathrm{kg} \mathrm{MS}$ de fourrage ingéré. 